\title{
Mixed carrier conduction in modulation-doped field effect transistors
}

\author{
S. E. Schacham, ${ }^{\text {a) }}$ E. J. Haugland, R. A. Mena, and S. A. Alterovitz \\ NASA Lewis Research Center, Cleveland, Ohis $4+1.35$
}

(Received 20 October 1994: accepted for publication 9 August 1995)

\begin{abstract}
The contribution of more than one carrier to the conductivity in modulation-doped field effect transistors (MODFET) affects the resultant mobility and complicates the characterization of these devices. Mixed conduction arises from the population of several subbands in the two-dimensional electron gas (2DEG), as well as the presence of a parallel path outside the 2DFG. We characterized GaAs/AlGaAs MODFET structures with both delta and continuous doping in the barrier. Based on simultaneous Hall and conductivity analysis we conclude that the parallel conduction is taking place in the AlGaAs barrier, as indicated by the carrier freezeout and activation energy. Thus, simple Hall analysis of these structures may lead to erroneous conclusions, particularly for real-life device structures. The distribution of the $2 \mathrm{D}$ electrons between the various confined subbands depends on the doping profile. While for a continuously doped barrier the Shubnikov-de Haas analysis shows superposition of two frequencies for concentrations below $10^{12} \mathrm{~cm}^{-2}$, for a delta doped structure the superposition is absent even at $50 \%$ larger concentrations. This result is confirmed by self-consistent analysis, which indicates that the concentration of the second subband hardly increases. () 1995 American Institute of Phusias
\end{abstract}

\section{NASA-TM-111701}

State-of-the-art electronic devices are mostly implemented in heterostructures, composed of materials of different physical properties. The two fundamental transport parameters, carrier concentration, and carrier mobility, are affected by the material composition and by the specific layered structure. Thus, these parameters must be determined experimentally for each and every structure. The determination of the onset of parallel conduction in modulation doped field effect transistors (MODFETs) is a key issue in both device and circuit design. ${ }^{\prime}$

The configuration of modern transistors and their implementation result most frequently in a conductive path which is not limited to a single layer or a single carrier but rather involves at least two types of carriers. The characterization of transport parameters is complicated when more than one carrier type is taking part in the conduction process. The conventional technique, based on a single carrier analysis of the longitudinal and Hall resistivities, gives inadequate results and may lead to erroneous conclusions.

Carrier concentrations of a two-dimensional electron gas (2DEG) can be determined most accurately by the Shubnikov-de Haas (SdH) effect. The trequency of oscillations of the longitudinal conductivity as a function of inverse magnetic field is linearly proportional to the concentration. However, while this technique is very accurate, it can be implemented only at liquid helium temperatures for carriers with relatively large mobilities and $2 \mathrm{D}$ concentrations typically above $1 \times 10^{11} \mathrm{~cm}^{2}$.

The mobility of the 2D electrons depends on their concentration. While the mobility increases with increased concentration, it suffers an abrupt drop as additional energy subbands are populated. ${ }^{2}$ Thus, the determination of the onset of the population of the second confined subband is significant

\footnotetext{
"National Rincurch Council-NASA Senior Research Associate, on leave from the Department of Electrical Engincering and Solid State Insitute, Techion-Isratel Inst, of Technolegy, Haifa 32000 , Isratel.
}

for the investigation of this mobility. At cryogenic temperature, the presence of a second populated subband generates a superposition of the two frequencies in the SdH wave form. ${ }^{3}$ The threshold for the population of the second subband is typically at a $2 \mathrm{D}$ concentration of about $7 \times 10^{11} \mathrm{~cm}^{-2}{ }^{4} \mathrm{In}-$ deed. measurements taken at about this concentration show an abrupt drop in mobility. ${ }^{5} \mathrm{SdH}$ wave forms recorded from samples with $2 \mathrm{D}$ concentrations of about $1 \times 10^{12} \mathrm{~cm}^{-2}$ show a very clear superposition of two frequencies. ${ }^{3}$

In order to determine the transport parameters in quantum structures in the presence of more than one carrier type we recently introduced the separation of carrier concentrations and mobilities based on the simultineous fit of the longitudinal and transverse magnetoresistivities. ${ }^{5}$ The two carrier separation was verified by the SdH results. Two more groups have followed with a similar approach based on the same technique, ${ }^{6.7}$ which indicates the significance of the present analysis to the research in the field. In thi kechnique. the experimental part requires the measurement of the longitudinal and transverse voltages as a function of magnetic field. The mathematical analysis involves the simultaneous fit of the classical magnetoresistance equations for two or more carriers. The fitting parameters are the concentrations and the mobilities of the various carriers. Their values are derived without any presumptions. Even though one group claims to use an all-analytical approach, ${ }^{8}$ they also utilize a fitting process to derive some intermediate parameters, and their method is essentially as numerical as are the other two.

One of the more significant results of our analysis ${ }^{5}$ is the experimental verification that the $2 \mathrm{DEC}$ concentration remains unchanged throughout the relevant temperature range, i.e., from liquid helium to room temperature. Even though this was predicted a decade ago," recent papers are still associating sheet carrier concentrations with results based on single carrier Hall analysis, showing an exponential increase of the sheet concentration with increasing temperatures 


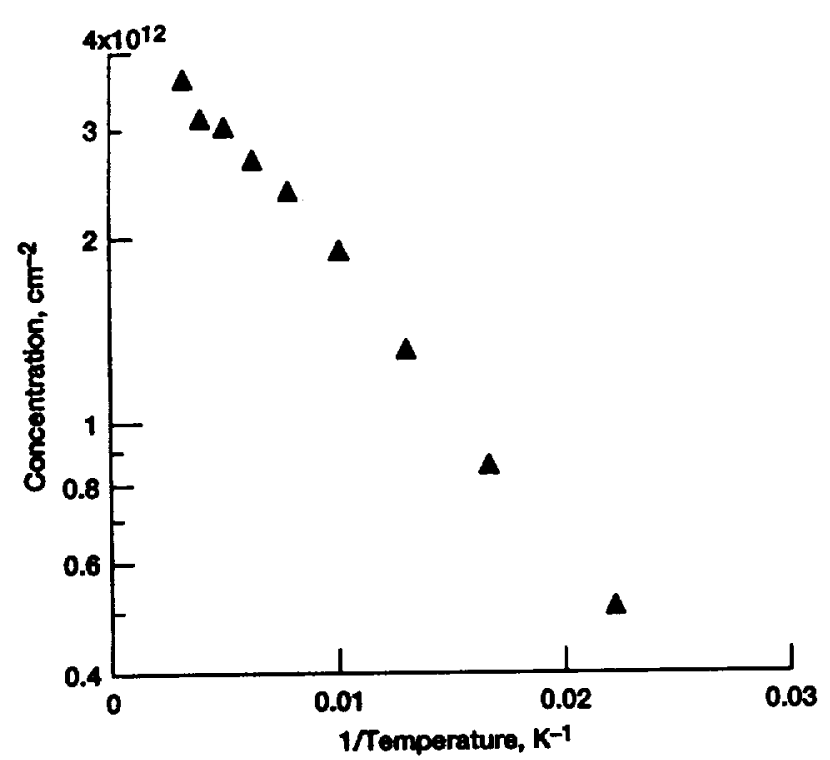

FIG. 1. Parallel carrier concentration vs inverse temperature derived from experimental data following carrier separation, showing freezeout with activation energy of 10 or $20 \mathrm{meV}$.

above about $150 \mathrm{~K} .^{10,11}$ Thus, by performing proper roomtemperature galvanomagnetic measurements ${ }^{5}$ it is possible to determine the low-temperature concentration.

A fundamental limitation of the two carrier analysis is that it is left to the researcher to determine how to associate the results with the various layers involved. This task may be complicated when the mobilities and concentrations are substantially different than anticipated. ${ }^{6}$ Even when there is a clear identification of the $2 \mathrm{D}$ carrier. there is still a debate which is the second, low mobility, carrier. This carrier is responsible for the apparent increase in concentration with increased temperature. Based on the separation of carriers, this parallel conduction was associated with the heavily doped GaAs cap layer, ${ }^{7.8}$ contradictory to the previous assumptions. $19.12-14$ The reasons given were that there was no clear indication of freezeout of these carriers, indicating a degenerate layer, and that etching off part of the cap layer resulted in reduction in the derived parallel concentration. However, a complete etching did not eliminate the parallel conduction but left a sheet concentration of close to $1 \times 10^{12} \mathrm{~cm}^{-2}$

Since the issue of parallel conduction is essential in MODFET device operation, we used the two carrier separation method in order to investigate the nature of the parallel carrier. Figure 1 shows the parallel (low mobility) carrier concentration as a function of inverse temperature as derived from galvanomagnetic measurements taken on a $\mathrm{Si}$ delta doped barrier of a GaAs/AlGaAs MODFET structure. The siructure was MBE grown by QED corporation and is described in detail elsewhere. ${ }^{15}$ The figure shows a clear freezeout of carriers in the parallel layer over more than an order of magnitude, indicating that the parallel conduction is not in a degenerate layer. The activation energy of this carrier is obtained from the slope of the dependence of the logarithm of the concentration versus $1 / T$. The derived activation

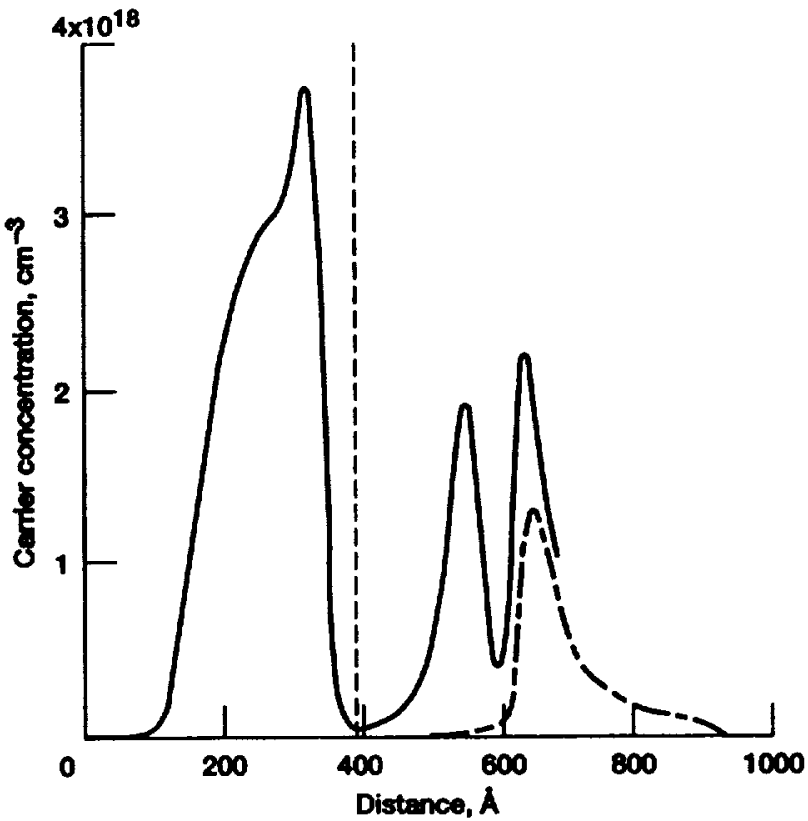

FIG. 2. Electron concentration derived from self-consistent simulation for delta-doped barrier with (solid line) and without (broken line) cap layer. The upper edge of the AIGaAs layer is marked by the vertical dashed line.

energy is about $20 \mathrm{meV}$ if no compensation is assumed, or $10 \mathrm{meV}$ otherwise. These values are within the range of ionization energy of the shallow Si donor in AlGaAs. ${ }^{16}$ On the other hand, the activation energy of silicon in GaAs is 5.8 $\mathrm{meV}$. Thus the clear exponential freezeout of parallel carriers to $40 \mathrm{~K}$ continuing ${ }^{15}$ all the way down to $20 \mathrm{~K}$ indicates that in our structures the second carrier derived from the analysis is not in the degenerate cap layer, but rather in the AlGaAs barrier. We obtained similar results in structures with a continuously doped barrier. Thus, we associate the parallel conduction with the barrier AlGaAs electrons.

The second argument for associating the parallel conducting with the cap layer was the clear reduction in its concentration with the etching of this layer. In order to clarify this matter we performed a self-consistent analysis of the structure. In this method, the Schrödinger and the Poisson equations are solved simultaneously, providing the proper wave functions and carrier concentration profile which satisfy both equations. In addition, the electric potential along with the energy-band diagram are derived. Measurements performed by Look et al. ${ }^{8}$ show a decrease in parallel concentration from $5.5 \times 10^{12}$ to $0.9 \times 10^{12} \mathrm{~cm}^{-2}$ following complete removal of the layer. On the other hand, as shown by the self-consistent analysis (Fig. 2), the removal of the cap layer also results in the annihilation of the barrier free electrons. The full line in Fig. 2 shows three peaks in concentration. The first, between 100 and $400 \AA$ represents the free electrons in the heavily doped cap layer. The second, between 500 and $600 \AA \AA$ shows the electrons in the barrier, which extends to $590 \AA$. The peak between 600 and $800 \AA$ is the 2DEG. The simulation for the structure without the cap layer is shown by the broken line. For clarity the origin was moved to 300$) \AA$, at the edge of the AlGatAs hartier. as indi- 




FIG. 3. Shubnikov-de Haas wave form tor a delta-doped barrier AICiad InGaAs MODFF:T structure, showing that only one subhand is poputalsol.

cated by the vertical dashed line. The only peak left is that of the 2DEG. Thus, the absence of parallel conduction following the removal of the GaAs cap layer proves that the parallel conduction is in either the cap or the barrier layers but not in any other layer such as the buffer GaAs." However, there is no insight as to whether it is located in the cap or ill the barrier. This present work clarifies this issue in a complete way.

It is well accepted that the onset of population of the second contined subband of the 2DEG in MODFET structures takes place at electron concentration of $7-8 \times 10^{11}$ $\mathrm{cm}^{2}$. This is manifested by an abrupt drop in mobility and by the appearance of a second frequency in the fast Fourier transform (FFT) of the recorded $\mathrm{SdH}$ waveform. Yet. our measurements on a structure with a delta doped AIGaAs barrier over an undoped GaAs did not show a superposilion with concentrations as high as $1.45 \times 10^{12} \mathrm{~cm}^{2}$ | see Fig. 4 (b) of Ref. 17]. Similarly Fig. 3 shows the $\mathrm{SdH}$ waveform

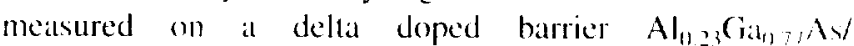
$\ln _{0.2} G_{a_{0} .8}$ As heterostructure with a well concentration of about $1.7 \times 10^{12} \mathrm{~cm}^{2}$. Indeed the $F F T$ shows a sillgle peith at $35.3 \mathrm{~T}$, equivalent to $1.7 \times 10^{12} \mathrm{~cm}^{2}$, as shown in the imet.

The calculation of the confined energy levels using selfconsistent analysis resolves this puogle. There is a importan difference between the energy bands in a continuously doped and a delta doped barrier. In the latter structures, as the carrier concentration increases the well is "pushed down" beneath the Fermi level $\left(E_{F}\right)$. As a result the ground level energy is further below $E_{l}$, with increased concentration." Even at populations as high as $1.7 \times 10^{12} \mathrm{~cm}^{-2}$ the concentration of the second subband is less than $3 \times 10^{11} \mathrm{~cm}^{-2}$. Moreover, the second confined wave function extends throughout the well areal, while the ground level is confined to its narrow section. Thus, the carriers in second energy level are more 3D in behavior, as a result their SdH oscillations are substantially weaker. Therefore, the superposition cannot be observed. It is interesting to note that in an early work, investigating continuously doped barriers, once a concentration of about $7 \times 10^{11} \mathrm{~cm}^{-2}$ was achieved, all additional carriers went into the excited subband. ${ }^{18}$ Here, in the delta doping case, the addition goes almost entirely to the ground level.

'S. Y. Chou, D. A. Antoniadis. and H. I. Smith. IFFE Trans. Electron Devices ED-34. 88.3 (1987).

'S. Mori and T. Ando. J. Phys. Sor. Jpn. 48, 86.5 (1980)

'S. L. Schacham, Ii. J. Haugland, and S. A. Alterovit\%. Pliys. Re'v, B 45 13417 (1992).

${ }^{4}$ A. Kastalsky and J. C. M. Hwang, Solid Statc Commun. 51. 317 11984t.

${ }^{5}$ S. E. Schacham. R. A. Menit. H. J. Hatuglind, and S. A. Altcrovite. Appl. Phys. I.ett. 62. 128.3 11993$\}$.

"J. S. Kim. D). (j. Seviler. and W. F. Tieng, J. Appl Phys, 7.3, 8.324 1199.31.

'D. C. Look, C. E. Stut, and C. A. Bozada. J. Appl. Phys. 74, 311 1199.3).

${ }^{x}$ D. C. look, B. Jogaii. C. E. Stutz. R. E. Sherril, (;. ('. DeSalvo. T. J. Rogers, and J. M. Ballingall. J. Appl. Phys. 76. 328 (1994)

"K. lee, M. S. Shur. T. J. Inrmmond. and H. Morkeg, I. Appl. Plyy. 54. (04.32 (1983).

"'J. Shen. S. Tehrani. H. Goronkin, R. Droopald, and G. Mallalcals. J. Appl. Phys. 71, 5985 (1092).

${ }^{11}$ K. Batlathatyya, J. O. Orwa. and S. M. Gondnick, J. Appl. Phys. 73, 4.3\% (1093).

"K. Lec. M. S. Shur. T. J. Drummond, and H. Morkeg. J. Appl. Phys. 54. $2093(1983)$.

"S. Hiyamiza, K. Nanhu, T. Mimura, T. Fujii, and H. Hashimolo, Jpu, J Appl. Phys. 20. I.378 (1081).

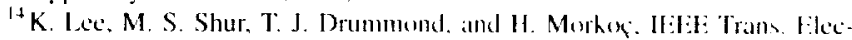
tron Devices ED-31, $88.3(1984)$.

ISS. E. Schacham, R. A. Mena, F. I. Hatugland, and S. A. Alterovile. J. Appl Phys. 78, 321 (1995).

"N. Chatnd, T. Hendersinn, J. Klem. W. T. Masseluik. R. Hischer. Y. C Chang. and H. Morkeş, Phys. Rev. B 30. 4481 (1984)

${ }^{17}$ R. A. Mena. S. E. Schacham, E. J. Haugland, and S. A. Alkerovil/. J. Appl. Phys. 74, 3973 (1093).

${ }^{18}$ H. 1. Stormer, A. C. Ciensard, and W. Wiegmimn, Solid Statc Commun. 41. 707110821 . 
